\title{
Downregulation of caspases and Fas ligand expression, and increased lifespan of neutrophils after transmigration across intestinal epithelium
}

\author{
G Le'Negrate ${ }^{1,5}$, P Rostagno $0^{2,5}$, P Auberger ${ }^{3}$, B Rossi ${ }^{1}$ \\ and $P$ Hofman ${ }^{\star, 1,4}$ \\ 1 Institut National de la Santé et de la Recherche Médicale (INSERM) U364, IFR \\ 50 Nice, France \\ 2 Laboratoire d'Oncogénétique, Centre Antoine Lacassagne, France \\ 3 INSERM U526, IFR 50 Nice, France \\ ${ }^{4}$ Laboratoire d 'Anatomie-Pathologique, Faculté de Médecine, Nice, France \\ 5 Current address: The Burnham Institute, La Jolla, CA 92037, USA \\ * Corresponding author: P Hofman, INSERM, FPI 02-15, Faculté de Médecine, \\ Avenue de Valombrose, 06107 Nice, Cedex 01, France. Tel: 33493 377759; \\ Fax: 33493 819456; E-mail: hofman@unice.fr
}

Received 11.4.02; revised 12.7.02; accepted 25.7.02

Edited by T Ferguson

\begin{abstract}
During inflammatory bowel diseases, commitment of extravased polymorphonuclear leucocytes (PMN) to apoptosis is required for the resolution of inflammation. To investigate the effect of transepithelial migration on PMN apoptotic rates, PMN transepithelial migration was reproduced in vitro using T84 intestinal monolayers. Transepithelial migration was found to delay neutrophil apoptosis, and this survival effect correlated with a downregulation of the surface expression of Fas ligand (FasL) and with a decrease in both procaspases-3, and -8 mRNA and procaspases $-3,-6,-7$ and -8 protein levels. Moreover, neutrophil survival and FasL shedding mediated by transepithelial migration were abrogated by a broad-spectrum metalloproteinase inhibitor, BB-94. Although Erk1/2 and p38 MAPK were activated in transmigrated PMN, inhibition of these MAP kinases did not impair transmigration-induced PMN survival. Taken together, our results show that transepithelial migration induces the downregulation of proapoptotic proteins expression in transmigrated PMN, which results in their increased lifespan.

Cell Death and Differentiation (2003) 10, 153-162. doi:10.1038/ sj.cdd. 4401110
\end{abstract}

Keywords: apoptosis; neutrophils; caspases; Fas; Fas ligand; migration

\footnotetext{
Abbreviations: DMEM, Dulbecco's modified Eagle's medium; ERK, extracellular signal-regulated kinase; FasL, Fas ligand; FMLP, formyl-methionine-leucine-phenylalanine; GM-CSF, granulocytes macrophage-colony stimulating factor; HBSS, Hank 's balanced salt solution; IL-, interleukin-; JNK, c-jun $\mathrm{NH}_{2}$-terminal kinase; LPS, lipopolysaccharides; MAPK, mitogen-activated protein kinase; PMN, polymorphonuclear leucocytes; TNF $\alpha$, tumor necrosis factor $\alpha$
}

\section{Introduction}

Acute inflammation of the intestine is characterized by polymorphonuclear leucocytes (PMN) migration first into the mucosa, then across the epithelium and ultimately into the intestinal lumen. ${ }^{1}$ Then, the efficiency of inflammatory response is limited by the PMN's short lifespan (within $72 \mathrm{~h}$ in blood stream), which die through the activation of an apoptotic program. ${ }^{2}$ PMN apoptosis and subsequent ingestion by macrophages are critical for their release of cytotoxic substances. ${ }^{2-7}$ However, several inflammatory mediators, such as host-derived cytokines IL1 , IFN- $\gamma$, GM-CSF, IL-8 or bacterial LPS have been shown to increase PMN lifespan. ${ }^{8-11}$ Conversely, this delayed apoptosis can be counterbalanced by others mediators such as TNF- $\alpha$, IL-10, activation of oxidative burst, or phagocytosis of some bacterial species. ${ }^{4,12-16}$ The transmigration process is initiated by the activation of neutrophils stimulated by inflammatory agents. Then, PMN migration requires sequential engagement of PMN adhesion molecules to their counter receptors on the endothelium, within the extracellular matrix and finally, on the epithelium. ${ }^{17,18}$ Activation of cell surface adhesion molecules during PMN recruitment has been shown to modulate apoptosis. For example, ligation of $\beta 2$ integrins during transendothelial migration delays neutrophil apoptosis. ${ }^{19}$ In contrast, some other studies have shown that aggregation and engagement of $\beta 2$ integrin by antibody crosslinking or during transendothelial migration could delay or promote apoptosis depending on the presence of proapoptotic stimuli. ${ }^{20,21}$ However, the role of transepithelial migration regulating PMN that are recruited to digestive lumen remained under investigation. Therefore, using T84 monolayers to mimic the intestinal epithelial barrier, we sought to investigate the effect of the transepithelial migration on PMN apoptotic rate.

The Fas (APO-1; CD95)/Fas ligand (FasL) system plays a crucial role in T-cell-mediated cytotoxicity, ${ }^{22}$ in the maintenance of immune privilege ${ }^{23}$ and regulating lymphocyte, ${ }^{24}$ eosinophil ${ }^{25}$ and monocyte ${ }^{26}$ homeostasis. In PMN, both Fas and FasL are constitutively expressed on the cell surface and therefore can induce cell death by both an autocrine or paracrine mechanisms, ${ }^{25}$ although previous studies suggested that spontaneous PMN cell death is not dependent on Fas-mediated signaling. ${ }^{28,29}$

PMN apoptotic pathways can be controlled by activation of mitogen-activated protein kinases (MAPK). Although p38 MAPK is activated in dying PMN, and could be required for activation of stress-induced PMN apoptosis, involvement of p38 MAPK in spontaneous and Fas-dependent apoptosis is still controversial. ${ }^{30-32}$ Albeit still discussed, the activation of Erk pathway has been shown to participate in 
the delay of constitutive apoptosis mediated by inflammatory mediators such as IL-8, LPS and GM-CSF. ${ }^{11,33}$

In this study, we show that transepithelial migration of PMN induced by IL-8 or FMLP delays PMN apoptosis. Increased survival of transmigrated $\mathrm{PMN}$ was associated with a decrease of surface FasL, and of procaspases-3, -6, -7 and -8 expression, while activation of Erk and p38 MAPK do not appear to be involved in transmigration-mediated PMN apoptotic protection.

\section{Results}

\section{Migration of PMN across intestinal epithelial monolayers delays their apoptosis}

To determine the effect of epithelial transmigration on PMN apoptosis, physiologically or nonphysiologically directed migration of PMN through T84 intestinal epithelial monolayers was induced either with IL- 8 or FMLP at $37^{\circ} \mathrm{C}$. Tunel assays were then used to evaluate the effect of the transmigration process on the kinetics of DNA fragmentation. Apoptotic rates of transmigrated PMN were compared with those obtained from nonmigrated PMN incubated in HBSS, IL-8 or FMLP in the absence of T84 monolayers. As shown in Figure 1a, induction of apoptosis was similar in cells incubated for $12 \mathrm{~h}$ in HBSS alone or in the presence of IL-8 or FMLP. In contrast, the extent of apoptosis in PMN (Tunel positive) that was transmigrated in response to either IL-8 or FMLP was significantly lower, regardless of the migration sense (physiological, or basolateral-to-apical versus nonphysiological, or apical-to-basolateral). These data suggest that transmigration induced an apoptotic delay in PMN that was independent of the polarization of the epithelial cells.

\section{Delay in PMN apoptosis correlates with a decreased expression of procaspases-8, $-3,-6$ and $-7$}

Considering the similar effect that IL-8 and FMLP exert on PMN apoptosis, we now used only IL-8 throughout the study.

To further investigate the mechanism underlying PMN survival, we assessed the expression of procaspases-3, -6, -7 and -8 after different treatments. As shown in Figure 2, the level of procaspase-8 expressed by transmigrated PMN was not modified for up to $6 \mathrm{~h}$, then abruptly declined by $12 \mathrm{~h}$. Interestingly, a rapid decrease in procaspase-8 was detected in PMN as soon as $4 \mathrm{~h}$ after exposure to $\mathrm{IL}-8$, which was comparable to the decrease in procaspase-8 induced by incubation in HBSS at $37^{\circ} \mathrm{C}$ (data not shown). The levels of procaspases-3, -6 and -7 were progressively decreased in PMN during their transmigration. Whereas the p17 active forms of caspases- 3 and -7 and the p11 active form of caspase- 6 could be detected in control PMN incubated in HBSS at $37^{\circ} \mathrm{C}$ (not shown), or during stimulation by IL-8, caspases remain uncleaved in transmigrated cells (Figure 2). Accordingly, and compared to control PMN kept in HBSS (-) at $4{ }^{\circ} \mathrm{C}$, a $6-10$-fold increase in caspase-3 activity was observed in control PMN incubated in $\mathrm{HBSS}$ at $37^{\circ} \mathrm{C}$, in

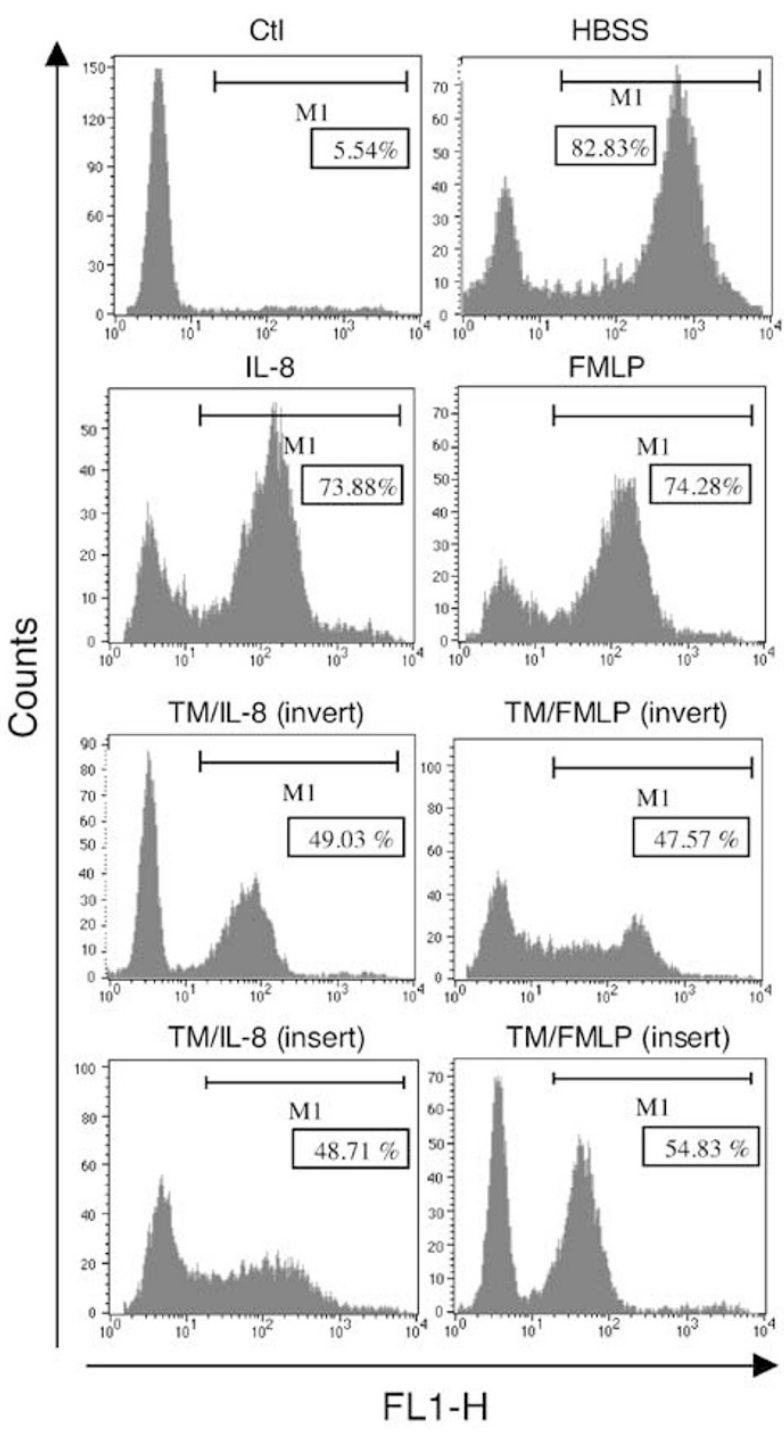

Figure 1 The apoptotic program of PMN is delayed during transmigration. (a) PMN were kept in HBSS $(-)$ at $4^{\circ} \mathrm{C}$ or were incubated either in HBSS alone (HBSS), IL-8 (IL-8) or FMLP (FMLP) during $12 \mathrm{~h}$ at $37^{\circ} \mathrm{C}$. Transmigration assays were performed through inserted monolayers (TM/LL-8 (insert)) or (TM/FMLP (insert)) for the nonphysiological direction, and through inverted monolayers (TM/ IL-8 (invert)) or (TM/FMLP (invert)) for the physiological direction. Tunel assays were performed to detect apoptotic PMN and the percentage of apoptotic cells are given for each condition (data are representative of at least three independent experiments)

IL-8-activated PMN, or in nontransmigrated PMN remaining in the upper chamber during $6 \mathrm{~h}$. This spontaneous caspase activation was dramatically reduced in transmigrated PMN (Figure 3a), and a similar profile of caspase-3 activity in PMN was obtained after $12 \mathrm{~h}$ of transmigration or treatment (Figure 3b).

To better delineate the mechanisms underlying the reduction on procaspases induced by PMN transmigration, mRNAs encoding caspases- 3 and -8 were quantitated by Northern blot analysis. As shown in Figure $3 c$, the basal level caspase-3 mRNA was already severely reduced after IL8 treatment, but it was almost undetectable in trans- 

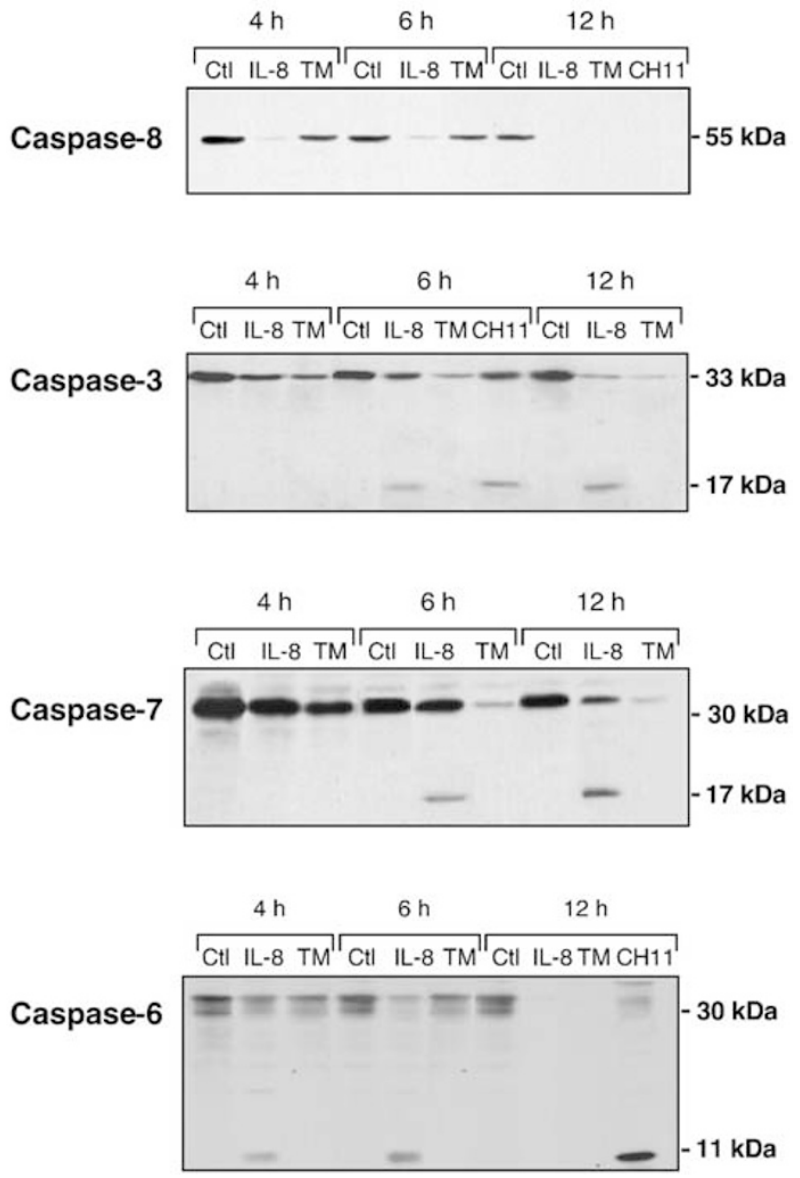

Figure 2 Transmigration induces a decrease in procaspases-8, $-3,-6$ and -7 expression in PMN. Immunoblot analysis of procaspases-8, -3, -7 and -6 activation during a time course of IL-8-induced transmigration. Profiles of procaspases expression in PMN control kept at $4^{\circ} \mathrm{C}$ in HBSS $(-)$ (Ctl) (to show the endogenous levels of caspases) or after transmigration assays (TM) were compared to those obtained after stimulation by IL-8 during the same period (IL8). Fas-induced apoptosis was used as positive control for caspases-8, -3 and -6 activation in PMN by incubation with an agonistic anti-Fas antibody $\mathrm{CH} 11(1 \mu \mathrm{g} /$ $\mathrm{ml}$; Immunotech) during indicated time (CH11). PMN supernatant lysates $(50 \mu \mathrm{g})$ were probed with antibodies to caspases-8, $-3,-7$ or -6 . Equal protein loading was confirmed by reprobing membranes with an $\mathrm{Ab}$ recognizing unphosphorylated p38 MAPK, as shown in Figure 6 (data are representative of at least three independent experiments)

migrated PMN. In contrast, caspase-8 mRNA was only slightly reduced in IL-8 treated PMN, but it was also undetected in PMN after $12 \mathrm{~h}$ of transmigration. As a loading control, glyceraldehyde-3-phosphate dehydrogenase (GAPDH) mRNA amounts were similar in PMN from all the three conditions (Figure 3c).

\section{FasL expression decreases in transmigrated PMN}

To characterize the survival mechanism involved in the antiapoptotic effect of transepithelial migration, Fas (CD95) and FasL expression were analyzed after PMN transmigration. As shown by flow cytometric analysis, transmigration failed to affect cell surface expression of Fas on PMN after $4 \mathrm{~h}$ of transmigration through T84 monolayer, whereas CD11b
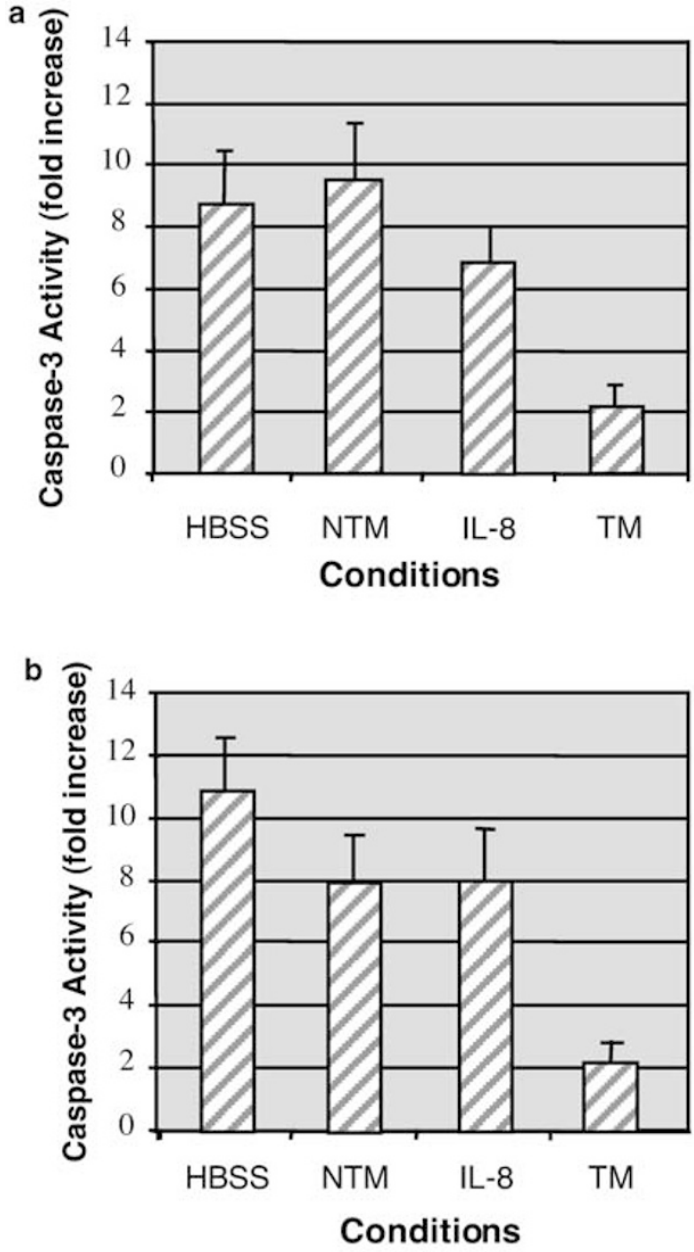

c CtI IL-8 TM JK

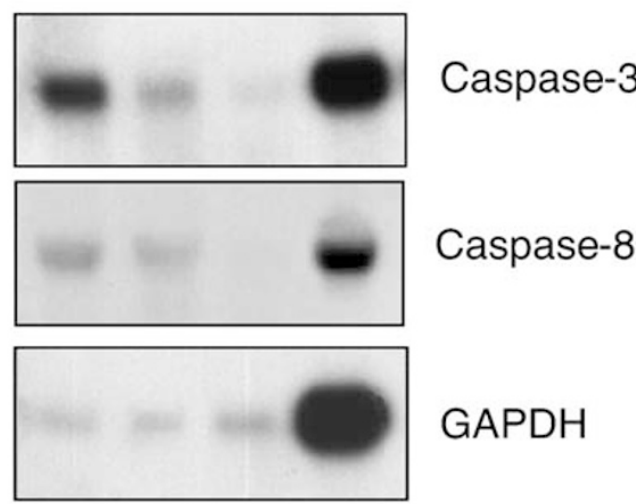

Figure 3 Caspase-3 activity assays were performed after $6 \mathrm{~h}(\mathbf{a})$ or $12 \mathrm{~h}(\mathbf{b})$ of transmigration assay (TM) or treatment. PMN were stimulated with $\mathrm{IL}-8$, incubated in HBSS at $37^{\circ} \mathrm{C}$ (HBSS), or collected from the upper chamber after the transmigration assay (NTM). Values obtained for each condition were compared to those obtained from PMN control kept in HBSS (-) at $4^{\circ} \mathrm{C}$, and reported as fold increase. The results represent the average value (+S.D.) from five independent experiments. (c) Northern blot analysis of caspases-3 and -8 mRNA expression in PMN control kept in HBSS $(-)$ at $4^{\circ} \mathrm{C}(\mathrm{Ctl})$, or after $12 \mathrm{~h}$ of IL-8-induced transmigration assay (TM), or after stimulation by IL-8 during the same period (IL-8). Expression of caspases-3 and -8 mRNA in Jurkat cells (JK) was used as a positive control. Membranes were stripped and reprobed with GAPDH probe to control for equal RNA loading in each condition (GAPDH) (data are representative of at least three independent transmigration assays) 
expression was upregulated, as we have previously shown in transmigrated PMN (Figure 4a). ${ }^{34}$ FasL expression by PMN was first investigated by reverse-transcriptase polymerase chain reaction (RT-PCR) assays. The expected $803 \mathrm{bp}$ amplicons were detected in PMN control, and in Jurkat cells

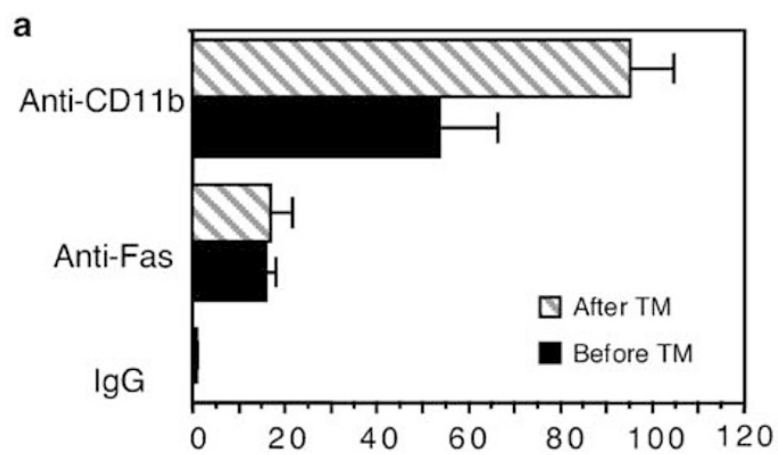

Mean fluorescence Intensity

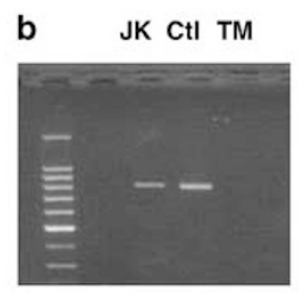

Fas-ligand

C

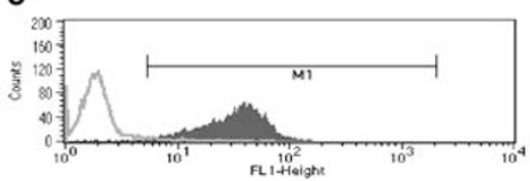

CtI
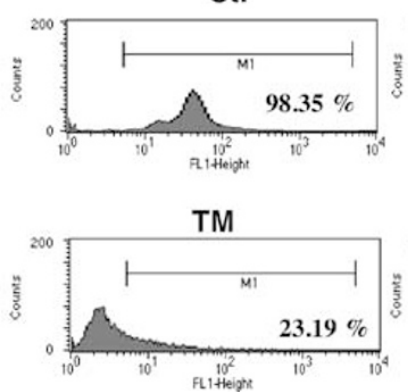

JK CtI TM

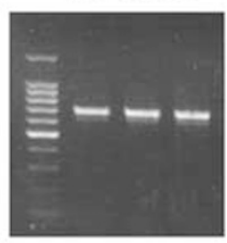

Actin

IL-8

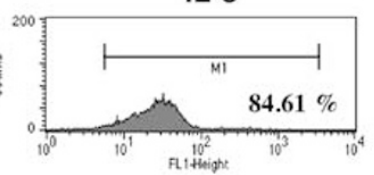

TM+BB94

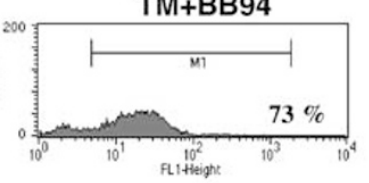

d

CtI

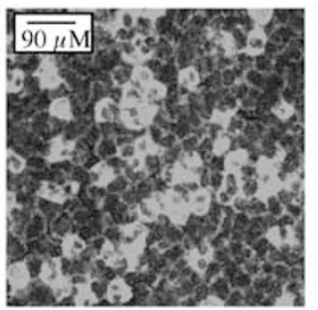

TM/Filters

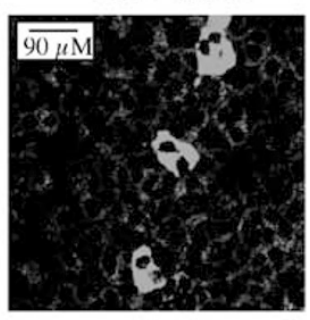

e
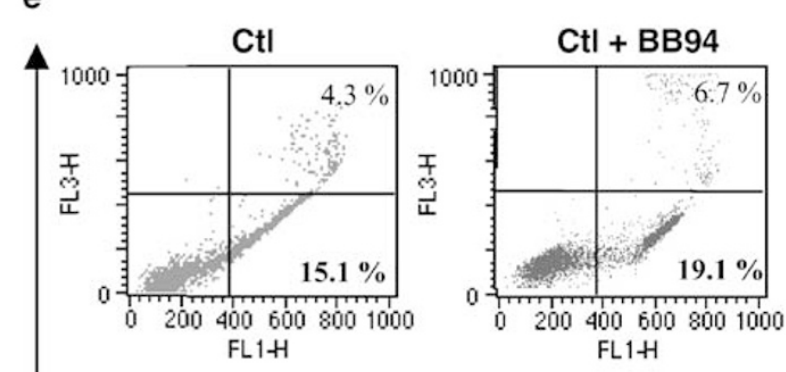

IL-8

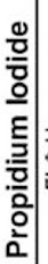

흠
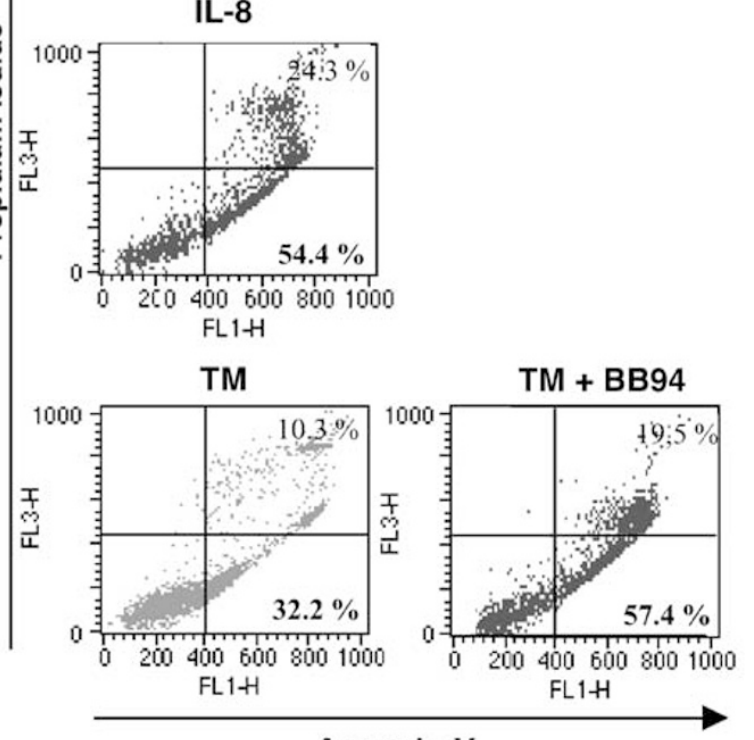

Annexin-V

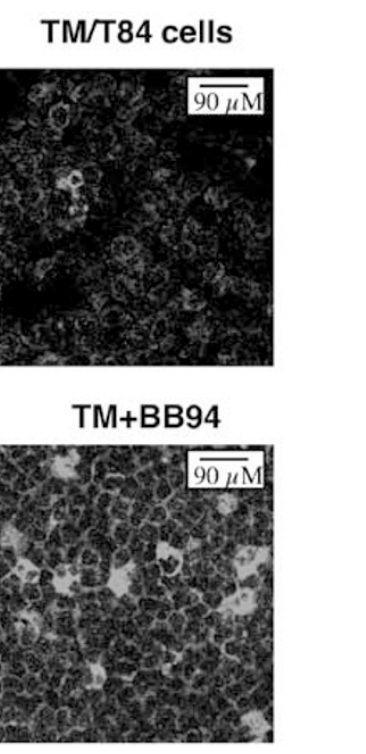

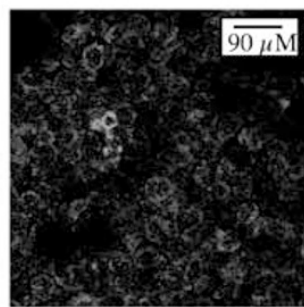

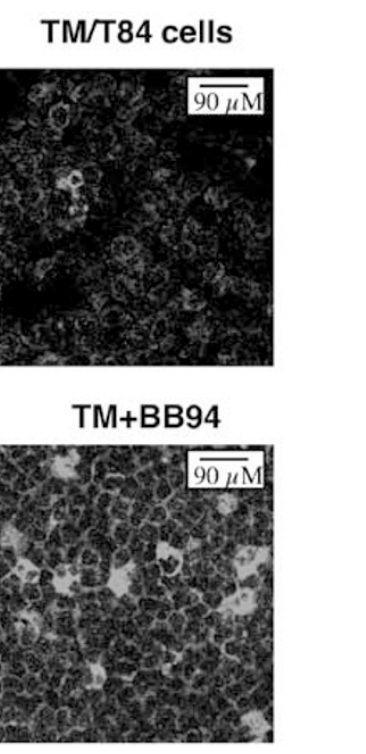

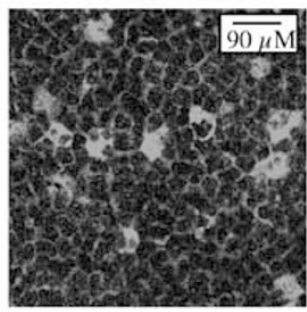
as positive control. In contrast, mRNA from PMN collected after $12 \mathrm{~h}$ of transmigration do not show FasL expression. Moreover, flow cytometric analysis indicated a decrease in FasL surface expression in transmigrated PMN, whereas IL-8

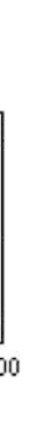

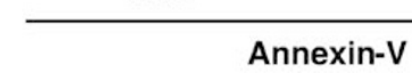


treatment alone did not affect FasL expression (Figure 4c). Previous reports have shown that FasL could be shed in a biologically active soluble form (sFasL) by an unidentified metalloproteinase..$^{35}$ In the aim to establish a putative causal relation between FasL cleavage and the antiapoptotic effect that transmigration exerts on PMN, two series of experiments were conducted. First, assessment of surface FasL expression by confocal microscopy revealed a similar decay in FasL expression on PMN subjected to migration through either an intestinal epithelial monolayer or an acellular filter (Figure 4d). Furthermore, as assessed by confocal microscopy and flow cytometry, transmigration-induced FasL decrease was abrogated in the presence of BB-94, a broad spectrum matrix metalloproteinase (MMP) inhibitor (Figure 4c, d). Then, the role of FasL cleavage in the survival effect of transmigration was addressed by preincubating PMN with BB-94 prior to a $12 \mathrm{~h}$ migration experiment. As shown in Figure 4e, FasL shedding was associated with a low apoptotic rate in transmigrated cells $(42.5 \%)$, whereas inhibition of FasL cleavage restored high apoptotic rate in transmigrated PMN (77\%). Altogether, these results underscore the involvement of FasL cleavage in the survival effect mediated by PMN transmigration.

\section{Fas receptor expressed by transmigrated PMN remains functional}

Since transmigration induces a decrease in caspases-3 and 8 , we investigated whether this downregulation process was associated with a reduced susceptibility to Fas-induced apoptosis in transmigrated cells. In this aim, PMN were treated with the anti-Fas $\mathrm{mAb} C H 11(1 \mu \mathrm{g} / \mathrm{ml})$ during $6 \mathrm{~h}$ after $12 \mathrm{~h}$ of transmigration or incubation in IL-8. As indicated by TUNEL assays, Fas induces apoptosis in both transmigrated and IL-8 stimulated cells. Although Fas remains functional after transmigration, caspases inhibition by ZVAD-fmk pretreatment fails to rescue transmigrated PMN from Fasinduced apoptosis (Figure 5a). Consistent with these results, caspases activity in transmigrated cells remains weak after treatment by $\mathrm{CH} 11$. Fas-induced apoptosis in transmigrated cells was not associated with caspases activation, indicating a caspase-independent apoptotic pathway. Opposite to these results, caspase-3 was activated during Fas-induced apoptosis in IL-8 stimulated cells.

\section{Activation of Erk and p38 MAP kinase is not involved in PMN apoptotic delay mediated by transepithelial migration}

As activation of Erk1/2 (p42/p44), p38 MAPK or Jun kinase (JNK) has been shown to be involved in the control of apoptosis, ${ }^{36}$ we investigated the effect of PMN transmigration process on MAP kinases activities. Time courses of different MAP kinases activation in PMN were followed by Western blot analysis using phospho-specific Abs to Erk1/2, p38 MAP kinases or JNK. As shown in Figure 6a, Erk 1/2 remained inactive in control cells, whereas IL-8 transiently activates Erk1/2 with a peak at $6 \mathrm{~h}$. Transmigration process increased the level of Erk1/2 phosphorylation in a sustained fashion for a least $12 \mathrm{~h}$ (Figure 6a). Activation of p38 and JNK was also detected in response to IL-8 in migratory and in non migratory conditions. Therefore, we investigated a possible causal relation between Erk, p38 MAPK and JNK activation and the control that transmigration exerts on PMN apoptosis. Specific inhibitors of these pathways were tested on PMN exposed to IL-8 in migratory or non migratory conditions. PMN were pretreated with PD98059 $(10 \mu \mathrm{M})$, SB203580 $(10 \mu \mathrm{M})$ or SB202190 $(10 \mu \mathrm{M})$ to respectively inhibit Erk1/2, p38 MAPK ( $\alpha$ and $\beta$ isoforms) or both p38 and JNK. As evidenced by Tunel DNA staining, the inhibition of Erk1/2, p38 MAPK and JNK was without any effect on the onset of the apoptotic rate in transmigrated PMN (Figure 6b). At variance with these results, inhibition of Erk1/2 of PMN exposed to IL-8 had no significant effect on the onset of their cell death, whereas inhibition of p38 MAP kinase activity delayed PMN apoptosis.

\section{Discussion}

Massive migration of PMN across the colonic epithelium constitutes the earlier effective response observed during bacterial colitis or in the acute phase of inflammatory bowel diseases. ${ }^{1}$ Accumulation of PMN in tissues could enhance the inflammatory response while the induction and control of neutrophil apoptosis, followed by their phagocytosis by macrophages is crucial for the resolution of the inflammation. $^{2-4}$ Although several inflammatory stimuli have been shown to control PMN apoptosis, the role of transepithelial migration on their apoptotic program remained to be determined. To address this question, we have used an

Figure 4 Fas (CD95) and FasL expression in PMN. (a) Untreated PMN (before TM) or after $4 \mathrm{~h}$ of transmigration assays (after TM) were analyzed for Fas (CD95) and CD11b surface expression by flow cytometry using specific mAb to Fas (ZB4, Immunotech) or to CD11b (OKM1, ATCC). CD11b staining was performed as a positive control for transmigration induced upregulation of cell surface adhesion receptor expression (CD11b). PMN were also stained with an irrelevant IgG as negative control $(\mathrm{lgG})$. Bars represent the average of mean fluorescence intensity from five independent experiments. (b) RT-PCR assays for FasL mRNA. Primers were designed to yield an 803 bp product. RNA from Jurkat cells stimulated with PMA served as positive control (JK). FasL mRNA expression was analyzed in PMN control kept at $4{ }^{\circ} \mathrm{C}$, or after $12 \mathrm{~h}$ of transmigration induced by IL-8 (TM). RT-negative amplifications showed no product in the positive control (data not shown), and equal cDNA loading was verified by amplifying actin mRNA. (c) Analysis of cell surface expression of FasL on PMN control kept in HBSS (-) buffer at $4{ }^{\circ} \mathrm{C}$ (Ctl), after $4 \mathrm{~h}$ of incubation in IL-8 (IL8), or after $4 \mathrm{~h}$ of transmigration either in the absence (TM), or in the presence of $10 \mu \mathrm{M}$ of BB-94 during $4 \mathrm{~h}$ of transmigration assay (TM+BB-94). Cells were stained with anti-human FasL (Santa Cruz Biotechnology) directed against an extracellular epitope of the molecule. The staining was then analyzed by flow cytometry. Staining with antibodies to CD11b was used as a positive control (CD11b) and irrelevant IgG as a negative control (IgG). Data are representative of three independent experiments. (d) Confocal analysis of FasL cleavage inhibition by a MMP inhibitor (BB-94). PMN control $\left(1 \times 10^{6}\right)$ (Ctl) or after $4 \mathrm{~h}$ of transmigration assay were stained with anti-FasL antibody (Santa Cruz Biotechnology) followed by secondary antibody linked to FITC. Staining of transmigrated PMN treated with BB-94 (TM+BB94) was compared to those obtained after transmigration through T84 cells (TM/T84 cells) or through acellular filters (TM/Filters). (e) Assessment of PMN apoptosis by annexin-V and propidium staining. Apoptotic cell population is reported as the addition of R1, the lower right area R1 (early apoptotic events), and R2 the upper right area R2 (late apoptosis). PMN were kept at $4^{\circ} \mathrm{C}$ in HBSS (-) in the absence (Ctl) or in presence of BB-94 (Ctl+BB94), or were stimulated by IL-8 (IL-8) during $12 \mathrm{~h}$. PMN were also used for an IL-8-induced transmigration assay, in absence (TM), or in presence of BB-94 (TM+BB94) for a total of $12 \mathrm{~h}$ (data are representative of at least three individual experiments) 


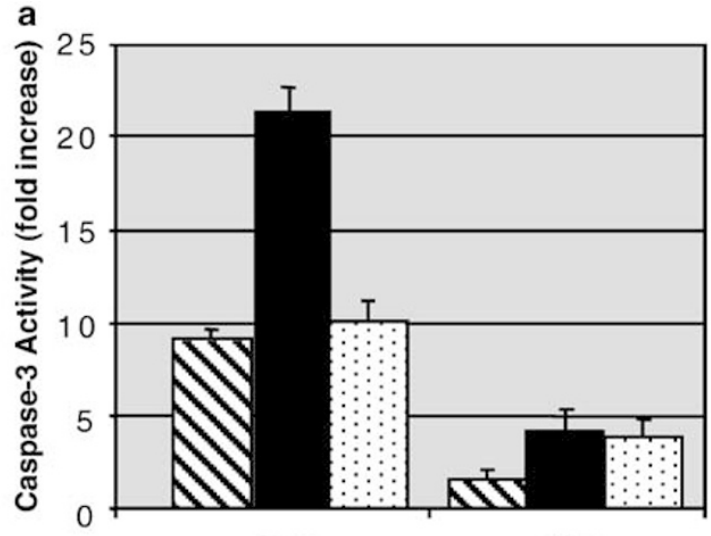

IL-8

TM

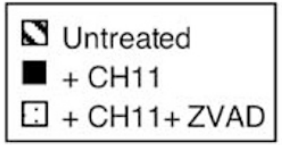

Conditions

b

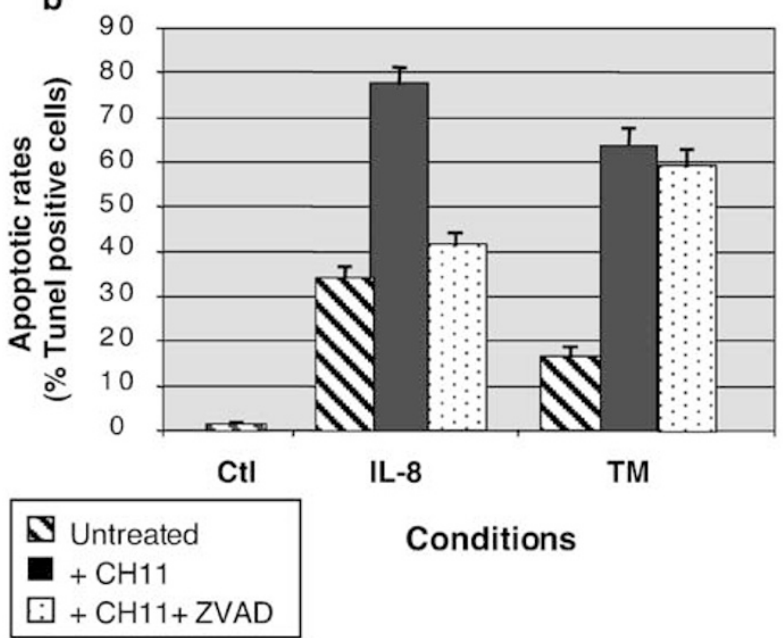

Figure 5 (a) Caspase-3 activity and (b) Tunel analysis of Fas-induced PMN apoptosis after transmigration. After $12 \mathrm{~h}$ of transmigration induced by IL-8 (TM), or incubation in IL-8 during the same period (IL-8), PMN were treated with $\mathrm{CH} 11$ antibody $(1 \mu \mathrm{g} / \mathrm{ml})$ during $6 \mathrm{~h}$. In the aim to investigate the involvement of caspases activation during Fas-induced apoptosis, PMN were preincubated with the fluoromethyl ketone inhibitor ZVAD-FMK (200 $\mu \mathrm{M}$; Alexis Corporation) for $2 \mathrm{~h}$ before transmigration assay (performed in presence of ZVAD-CHO) and $\mathrm{CH} 11$ treatment. (a) Caspase-3 activity obtained for each condition was compared to those obtained from PMN control kept in HBSS $(-)$ at $4^{\circ} \mathrm{C}$, and reported as fold increase. (b) Tunel assays were performed to detect apoptotic PMN and percentage of apoptotic cells are given for each condition. The results are representative of the average value (+S.D.) from three experiments

in vitro model that mimics massive PMN transepithelial migration. By using Tunel DNA staining, we found that the transepithelial migration process by itself prolonged the PMN lifespan regardless the chemoattractant used (IL-8 or FMLP). Since we have previously shown that most of the PMN added in the upper chamber migrate across T84 cells after $12 \mathrm{~h}$ of experiment, this survival effect of the transmigration process cannot be accounted to the selection of a longer-lived PMN subpopulation. ${ }^{37}$ Moreover, transmigration-mediated neutrophil survival could not be because of contact with T84 cells or
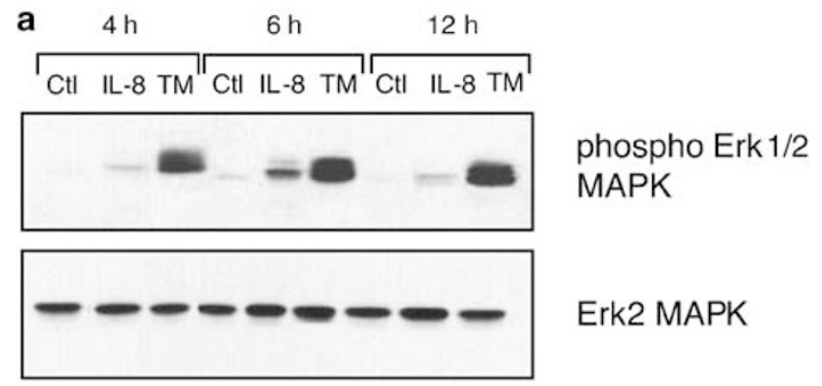

\section{Erk2 MAPK}
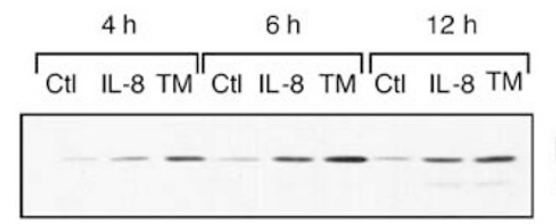

phospho p38 MAPK

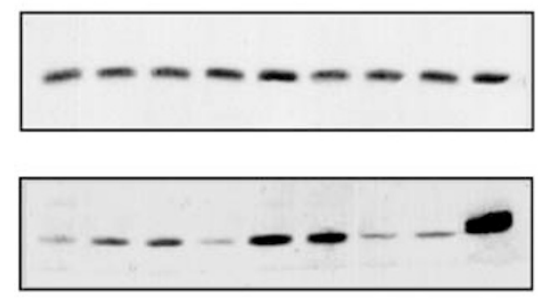

p38 MAPK

phospho JNK

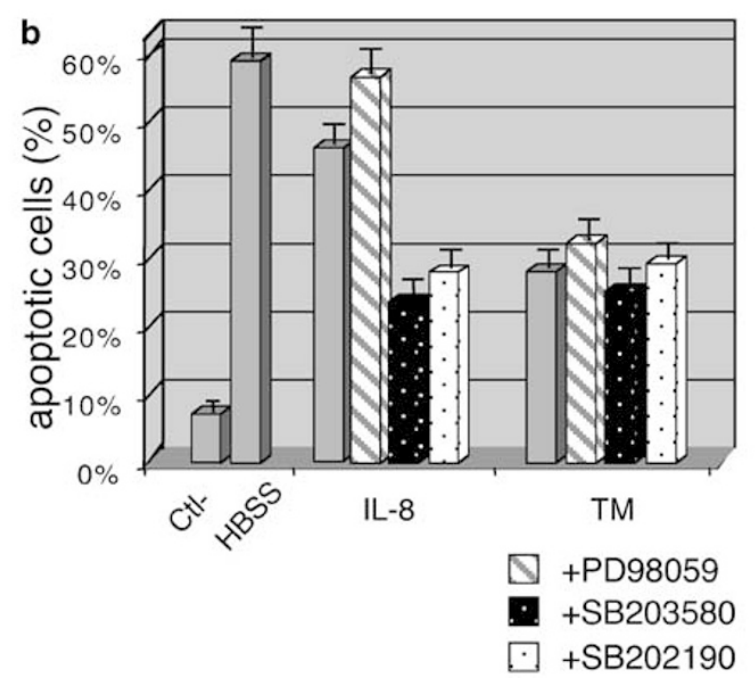

Figure 6 Activation of Erk and of p38 MAPK is not involved in the transmigration-induced PMN survival. (a) Effects of transmigration on Erk, p38 MAPK and JNK kinase activation in PMN were analyzed by immunoblotting using Abs against phosphorylated forms of Erk1/2, p38 MAPK and JNK. During the indicated periods, control PMN were kept at $4^{\circ} \mathrm{C}$ in HBSS (-) (Ctl), stimulated with IL-8 (IL-8), or collected after $4-12 \mathrm{~h}$ of transmigration assays (TM) as indicated. Equal protein loading was confirmed by reprobing membranes with Abs recognizing Erk2, and p38 MAPK irrespective of their status of phosphorylation. (b) Apoptotic PMN were detected by Tunel DNA staining and flow cytometric analysis after $12 \mathrm{~h}$ of treatment or transmigration. PMN were kept in HBSS $(-)$ at $4^{\circ} \mathrm{C}(\mathrm{Ctl})$, or in $\mathrm{HBSS}$ at $37^{\circ} \mathrm{C}$ (HBSS). PMN were also stimulated with IL-8 (IL-8), or used for transmigration assays (TM). At $2 \mathrm{~h}$ before IL-8stimulation, or before the transmigration assays, Erk, p38 or both p38 and JNK were inhibited by preincubating PMN in PD98059 (20 $\mu \mathrm{M}$; Calbiochem) in SB203580 (10 $\mu \mathrm{M}$; Calbiochem) and in SB202190 (10 $\mu \mathrm{M}$, Calbiochem) respectively (data shown are representative of five independent experiments) 
to antiapoptotic cytokines secreted by these cells. First, the sole contact of PMN with T84 cells in the presence of IL-8 did not prevent caspase- 3 activation and spontaneous apoptosis (unpublished data). Second, only small amounts of antiapoptotic cytokines such as GM-CSF and IL-1 $\beta$ were detected in transmigration medium, and neutralization of these cytokines with specific antibodies had no effect on PMN apoptosis (unpublished data). Although the exact role played by enterocytes in PMN survival remains to be elucidated, our findings indicate that the transepithelial migration process itself is necessary to delay PMN apoptosis. Consistent with our results, previous data have shown a prolonged lifespan of extravased $\mathrm{PMN}$ following either in vitro transmigration across endothelial barrier or in vivo transmigration into the lung. ${ }^{19,38}$ This prolonged PMN lifespan is likely to be associated with an increased functionality, since we have previously shown an increased phagocytosis of Escherichia coli by transmigrated PMN. ${ }^{34}$

To further characterize the survival mechanisms involved in the antiapoptotic effect of transepithelial migration, we have investigated the role of the Fas/FasL pathway in PMN in this process. In accordance with previous studies, our results clearly indicate that PMN constitutivelly express FasL. ${ }^{27,39}$ Furthermore, whereas Fas (CD95) expressed on PMN remained unaltered, FasL was rapidly cleaved during transmigration. Consistent with these findings, previous data have shown the presence of a high level of soluble FasL (sFasL) in serum from patients with inflammatory diseases. ${ }^{40}$ Moreover, the present work provides evidence suggesting that a decrease in FasL on PMN was involved in the antiapoptotic effect of transmigration, probably by preventing both paracrine and autocrine interactions between Fas and FasL on PMN surface. In this regard, the metalloproteinase inhibitor BB-94 prevented FasL shedding and abrogated the antiapoptotic effect of PMN transepithelial migration. It is interesting to note that pretreatment of T84 cells with the metalloproteinase inhibitor BB-94 did not affect FasL shedding in transmigrated PMN, suggesting that activation of PMN metalloproteinase(s), but not T84-metalloproteinases, is responsible for FasL cleavage (unpublished data). We have also investigated the role of caspase activation in regulating apoptosis of transmigrated PMN. We have found that in non migrating conditions, caspase-8 activation was observed first and it was followed by caspases- 3 and -7 activation. These data are consistent with previous studies showing that active caspase- 8 could trigger an amplification caspase activation cascade. $^{41}$

Interestingly, we observed that in transmigration conditions, there is a pronounced decrease in procaspases-8, $-3,-6$ and 7 expression that also correlated with a reduced caspases- 3 and -8 mRNA expression. Given the crucial role of caspases-8 and -3 activation in the induction and execution of neutrophil apoptosis, ${ }^{41,42}$ we hypothesize that transmigration-mediated downregulation of caspases accounts for the resistance to apoptosis of extravased PMN. Nonetheless, despite of this decrease in caspase expression and activity, Fas receptor is still functional and induces apoptosis in transmigrated cells. Consistent with downregulation in caspases expression, caspase inhibition fails to impair Fas-induced apoptosis supporting the evidence of a caspase-independent apoptotic process in transmigrated cells. However, further studies will be needed to characterize the molecular events involved in these processes. Consistent with our findings, the apoptotic delay observed in PMN isolated from the inflammatory sites or from patients with inflammatory bowel diseases was also associated with reduced expression levels of caspases. $^{36,43,44}$

MAP kinases have been implicated in the regulation of apoptosis. ${ }^{36}$ In our conditions, the Erk pathway was activated in response to IL-8 and was enhanced by the transmigration process. However, inhibition of Erk by the specific inhibitor PD98059 failed to affect the transmigration-mediated apoptotic delay in PMN. These results are in agreement with previous data indicating that activation of PMN spontaneous apoptosis was Erk independent. ${ }^{11,30,45}$

Similarly, p38 MAPK and JNK activation induced by IL-8 was also enhanced by transmigration, but inhibition of these enzymes did not modify the onset of apoptosis in transmigrated PMN. These results indicate that p38 MAP kinases are not involved in the antiapoptotic effect of the transepithelial migration. In contrast, inhibition of p38 MAPK and JNK activation in non migrating conditions strongly diminished the PMN apoptotic rate, suggesting that activation of these enzymes is required for neutrophil spontaneous apoptosis. The apoptosis-promoting effect of p38 activity in PMN spontaneous apoptosis has also been reported by Aoshiba et al., ${ }^{31}$ which have observed that p38 MAPK was constitutively active during spontaneous apoptosis, and that inhibition of this enzyme delayed this process.

In summary, our data provide the first evidence that transepithelial migration of activated PMN hinders their programmed cell death by inducing FasL shedding. This increased PMN survival was also correlated with a downregulation of caspases expression in transmigrated cells. By increasing PMN survival and function, the transepithelial migration process facilitates the adaptive response to infectious challenge. ${ }^{34}$ Nevertheless, during the acute phase of inflammatory diseases, which is characterized by massive PMN recruitment, the downregulation of proapoptotic proteins in transmigrated PMN might contribute to their accumulation, hence leading to tissue injury and development of chronic inflammation. ${ }^{1,2,17,46}$

\section{Materials and Methods}

\section{PMN isolation and cell culture}

Human PMN were isolated from whole blood using a Ficoll gradient followed by gelatin sedimentation. Residual erythrocytes were lysed with isotonic ammonium chloride and PMN were washed in HBSS (-) (without $\mathrm{Ca}^{2+}$ and $\mathrm{Mg}^{2+}$ ) (Sigma-Aldrich), as previously described. ${ }^{34,47}$ PMN (95\% pure) with $98 \%$ viability by trypan blue exclusion were resuspended in HBSS (-) and kept at $4^{\circ} \mathrm{C}$ before being used within $1 \mathrm{~h}$ after isolation.

The human colonic carcinoma cell line, T84 (passages 60-90) was obtained from the American Type Culture Collection (ATCC, Rockville, MD, USA). T84 cells were grown and maintained as confluent monolayers on collagen-coated permeable supports in medium (1:1 mixture of DMEM and Hanks F-12), supplemented with $15 \mathrm{mM}$ HEPES buffer ( $\mathrm{pH} 7.5)$, $14 \mathrm{mM} \mathrm{NaHCO} 3,40 \mathrm{mg} / \mathrm{ml}$ penicillin, $90 \mathrm{mg} / \mathrm{ml}$ streptomycin, $8 \mathrm{mg} / \mathrm{ml}$ of ampicillin and $5 \%(\mathrm{v} / \mathrm{v})$ heat-inactivated FBS. Inverted monolayers were 
grown on collagen-coated $0.33 \mathrm{~cm}^{2}$ ring-supported polycarbonate filters (Costar, Cambridge, MA, USA) and inserted monolayers were grown on collagen-coated $5 \mathrm{~cm}^{2}$ filters. Monolayers were utilized $6-10$ days after plating as previously described. ${ }^{48}$

\section{Transmigration assay}

PMN transmigration assays were performed at $37^{\circ} \mathrm{C}$ as previously described using low-attachment Costar plates (Cambridge, MA, USA). ${ }^{17,47,49}$ T84 cells were seeded on filters and were washed in HBSS (Sigma-Aldrich) before PMN transmigration assays. To allow a chemotactic gradient agent to form before transmigration assays, $100 \mathrm{ng} / \mathrm{ml} \mathrm{IL-8}$ or $10^{-7} \mathrm{M}$ FMLP in HBSS was applied in the lower chamber $15 \mathrm{~min}$ before adding PMN to the upper chamber. For basolateral-to-apical (physiological sense) directed PMN-transmigration assays, $5 \times 10^{6}$ PMN $\left(25 \times 10^{6}\right.$ cells $\left./ \mathrm{ml}\right)$ were added in the upper chambers of inverted monolayers grown on $0.33 \mathrm{~cm}^{2}$ filters. For the apicalto-basolateral (nonphysiological sense) directed transmigration assays, $15 \times 10^{6} \mathrm{PMN}\left(40 \times 10^{6} \mathrm{cells} / \mathrm{ml}\right)$ were added in the upper chambers of inserted monolayers grown on $5 \mathrm{~cm}^{2}$ filters. ${ }^{47}$ Since apical-to-basolateral transmigration allowed more PMN to be harvested, this pathway was used for biochemical experiments. The number of PMN that transmigrated into the lower chambers were assayed by quantification of azurophil granule marker myeloperoxidase (MPO), as previously described. ${ }^{37,47,49}$

As negative control, PMN $\left(2 \times 10^{6} / \mathrm{ml}\right)$ were kept in HBSS(-) at $4^{\circ} \mathrm{C}$, or were incubated in ultra-low-attachement Costar plates in the presence of $100 \mathrm{ng} / \mathrm{ml} \mathrm{IL-} 8$ or in $10^{-7} \mathrm{M}$ FMLP diluted in HBSS.

The MMP inhibitor BB-94 was kindly provided by British Biotech. This agent $(10 \mu \mathrm{M})$ was added to $\mathrm{PMN}$ for $1 \mathrm{~h}$ before transmigration assays and diluted in the transmigration medium during transmigration assays.

\section{TUNEL assay, FITC-labeled annexin-V and propidium iodide staining to detect apoptosis}

TUNEL assays were performed using the In Situ Cell Death Detection Kit (Roche, Mannheim, Germany), following the manufacturer's protocol. After staining, mean fluorescence intensity (MFI) was quantified on 20000 cells using a FACScan ${ }^{\mathrm{TM}}$ flow cytometer (Becton Dickinson, Rutherford, NJ, USA).

Briefly, PMN $\left(10^{6}\right)$ were incubated with fluorescein isothiocyanate (FITC)-labeled annexin-V (Roche) diluted in HNS buffer (10 mM HEPES$\mathrm{NaOH}$ (pH 7.4), $140 \mathrm{mM} \mathrm{NaCl}, 5 \mathrm{mM} \mathrm{CaCl}_{2}$ ) for 30 min at $37^{\circ} \mathrm{C}$ in the dark, as specified by the manufacturer. Propidium iodide $(10 \mu \mathrm{g} / \mathrm{ml})$ was added to the cell suspension just before analysis by flow cytometer.

\section{Detection of Fas and FasL cell surface expression}

A total of $10^{6}$ PMN were suspended in blocking buffer (PBS, $0.1 \mathrm{mg} / \mathrm{ml}$ human $\mathrm{lg}, 0.1 \% \mathrm{BSA}(\mathrm{v} / \mathrm{v}), 10 \mu \mathrm{g} / \mathrm{ml}$ goat serum) and incubated for $30 \mathrm{~min}$ at $4^{\circ} \mathrm{C}$. Then cells were stained using anti-Fas antibody (ZB4, $10 \mu \mathrm{g} / \mathrm{ml}$; Immunotech, Luminy, France), or anti-FasL antibody $(10 \mu \mathrm{g} / \mathrm{ml}$; Santa Cruz Biotechnology, Inc), followed by incubation with FITC-labeled rabbit anti-mouse or goat anti-rabbit secondary antibody (dilution 1:20; Dako, Glostrup, Denmark). Cells were then whashed in PBS and fixed in $0.4 \%$ formaldehyde. At least 10000 cells per sample were analyzed on a FACScan $^{\text {TM }}$ flow cytometer (Becton Dickinson). PMN were stained with anti-CD11b (OKM1, ATCC) or with isotype-matched control antibodies as positive and negative controls, respectively.

\section{Immunoblot analysis}

Briefly, PMN were washed in PBS and resuspended in ice-cold lysis buffer (10 mM HEPES, $3.5 \mathrm{mM} \mathrm{MgCl}_{2}, 150 \mathrm{mM} \mathrm{NaCl}, 1 \% \mathrm{NP} 40,1 \mathrm{mM} \mathrm{Na}_{3} \mathrm{VO}_{4}$, $1 \mathrm{mM}$ PMSF, $25 \mu \mathrm{M}$ leupeptin, $5 \mathrm{mM}$ benzamidine, $1 \mu \mathrm{M}$ pepstatin, $25 \mu \mathrm{M}$ aprotinin, $50 \mathrm{mM}$ sodium $\beta$-glycerophosphate, $20 \mathrm{mM} \mathrm{NaF}, 0.5 \mathrm{mM}$ DTT; all from Sigma-Aldrich). ${ }^{37}$ Protein lysates $(50 \mu \mathrm{g})$ were subjected to SDSPAGE and subsequently electrophoretically transferred onto nitrocellulose membranes. The membranes were incubated in blocking buffer and then probed with the primary antibody overnight at $4^{\circ} \mathrm{C}$. After incubation with peroxidase conjugated secondary antibody (rabbit anti-mouse Ig, dilution $1: 5000$ or goat anti-rabbit $\mathrm{lg}$, dilution 1:10.000; both from Dako), antigens were visualized by enhanced chemiluminescence detection (ECL kit, Amersham Pharmacia Biotech). The antibodies used were anti-Erk2 (Santa Cruz Biotechnology, Inc.), anti-phospho Erk1/Erk2 (New England Biolabs Inc.); anti-phospho p38 MAP kinase (Thr180/Tyr182) (New England Biolabs Inc.); anti-p38 MAP Kinase (New England Biolabs Inc); anti-SAP Kinase (New England Biolabs Inc); polyclonal anti-phospho Jun Kinase (Promega corp.); anti-caspase-8 (N-19) (IgG isotype clone obtained from Santa Cruz Biotechnology, Inc.) all diluted at 1:1000. The antibodies to caspase-6 (IgG1 isotype clone B93-4 obtained from BD PharMingen), caspase-3, (IgG2a isotype clone 19 purchased from Transduction Laboratories) and caspase-7 (IgG1 isotype clone B94-1 obtained from Transduction Laboratories) were used at $0.25-1 \mu \mathrm{g} / \mathrm{ml}$.

\section{DEVD-PNA cleavage assay}

Caspase activity was measured using a colorimetric assay. Briefly, PMN were resuspended in PBS containing $2 \mathrm{mM}$ DTT at $4^{\circ} \mathrm{C}$. After sonication for $8 \mathrm{~s}$ bursts, cells lysates $(100 \mu \mathrm{g})$ were incubated with $200 \mu \mathrm{M}$ of AcetylAsp-Glu-Val-Asp-pNA (Ac-DEVD-pNA) (Alexis Corporation, San Diego, CA, USA) which is preferentially cleaved by caspase-3. Release of pNA was monitored continuously at $37^{\circ} \mathrm{C}$ by using an excitation wavelength of $410 \mathrm{~nm}$ and specificity of the caspase assay was controlled by adding to cell extracts an apopain/CPP-32 inhibitor (DEVD-CHO) $(100 \mu \mathrm{M})$ (Alexis Corp.). Results were expressed as $\mathrm{pmol} / \mathrm{min} / \mathrm{mg}$ protein.

\section{RNA extraction, Northern blot analysis and RT-PCR assays}

PMN were washed in HBSS (-) buffer before total RNA was extracted by TRIzol reagent (Gibco) according to the manufacturer's instruction. RNAs (15 $\mu \mathrm{g} / \mathrm{sample}$ ) were fractionated by electrophoresis in a $1 \%$ agarose gel containing $1.2 \%$ formaldehyde, transferred onto a Hybond- $\mathrm{N}^{+}$nylon membrane, and hybridized sequentially with ${ }^{32} \mathrm{P}$-labeled DNA probes specific for procaspase-3, procaspase-8 or glyceraldehyde-3-phosphate dehydrogenase (GAPDH) at $42^{\circ} \mathrm{C}$ overnight. FasL expression was controlled by performing RT-PCR. Total RNA was treated with RNAsefree DNAse I and reversely transcribed using a SuperScipt ${ }^{\mathrm{TM}}$ Reverse Transcriptase (Life Technology, Gaithersburg, MD, USA). PCR was then performed from CDNA using the following primers pairs, respectively: human CD95L: 5'-ATGCAGCAGCCCTTCAATTAC-3' and 5'-TTGACCAGAGAGAGCTCAGAT- $3^{\prime}$. PCR products were loaded on a $1.2 \%$ agarose gel and vizualized with ethidium bromide. The expected length of the amplicons is $803 \mathrm{bp}$.

\section{Statistical analysis}

Statistical significance was determined using Student's $t$-test, and values were expressed as the mean and S.E.M. of $n$ number of experiment. 


\section{Acknowledgments}

This work was supported by Institut National de la Santé et de la Recherche Médicale (INSERM). We thank Ms D Sadoulet, Ms M Mari for their technical assistance, Mr. JC Chambard, and Mr. R Rezzonico for advices.

\section{References}

1. Haslett C, Savill JS, Whyte MKB, Stern M, Dransfield I and Meagher LC (1994) Granulocyte apoptosis and the control of inflammation. Phil. Trans. R. Soc. Lond. 345: 327-333

2. Haslett C (1992) Resolution of acute inflammation and the role of apoptosis in the tissue fate of granulocytes. Clin. Sci. 83: 639-648

3. Savill JS, Wyllie AH, Henson JE, Walport MJ, Henson PM and Haslett C (1989) Macrophage phagocytosis of aging neutrophils in inflammation: programmed cell death in the neutrophil leads to its recognition by macrophages. J. Clin. Invest. 83: 865-875

4. Cox G, Crossley J and Xing Z (1995) Macrophage engulfment of apoptotic neutrophils contributes to the resolution of acute pulmonary inflammation in vivo. Am. J. Resp. Cell. Mol. Biol. 12: 232-237

5. Savill J (1997) Apoptosis in resolution of inflammation. J. Leukoc Biol. 61: 375380

6. Wize J, Sopata I, Smerdel A and Maslinski S (1998) Ligation of selectin L and integrin CD11b/CD18 (mac-1) induces release of gelatinase B (MMP-9) from human neutrophils. Inflamm. Res. 47: 325-327

7. Akgul C, Moulding DA and Edwards SW (2001) Molecular control of neutrophil apoptosis. FEBS Lett. 487: 318-322

8. Lee A, Whyte MKB and Haslett C (1993) Inhibition of apoptosis and prolongation of neutrophil functional longevity by inflammatory mediators. J. Leukoc Biol. 54: 283-288

9. Watson RWG, Rotstein OD, Parodo J, Bitar R and Marshall JC (1998) The IL$1 \beta$-converting enzyme (caspase-1) inhibits apoptosis of inflammatory neutrophils through activation of IL-1 $\beta$. J. Immunol. 161: 957-962

10. Coxon A, Tang T and Mayadas TN. (1999) Cytokine-activated endothelial cells delay neutrophil apoptosis in vitro and in vivo: a role for granulocyte/ macrophage colony-stimulating factor. J. Exp. Med. 190: 923-933

11. Klein JB, Buridi A, Coxon PY, Madhavi JR, Manning T, Kettritz R and McLeish KR (2001) Role of extracellular signal-regulated kinase and phosphatidylinositol-3 kinase in chemoattractant and LPS delay of constitutive neutrophil apoptosis. Cell Signal. 13: 335-343

12. Cox G (1996) IL-10 enhances resolution of pulmonary inflammation in vivo by promoting apoptosis of neutrophils. Am. J. Physiol. 271: L566-L571

13. Watson GW, Redmond HP, Wang JH, Condron C and Bouchier-Hayes D (1996) Neutrophils undergo apoptosis following ingestion of Escherichia coli. J. Immunol. 156: 3986-3992

14. Lundqvist-Gustafsson $\mathrm{H}$ and Bengtsson $\mathrm{T}$ (1999) Activation of the granule pool of the NADPH oxidase accelerates apoptosis in human neutrophils. J. Leukoc Biol. 65: 196-204

15. Rotstein D, Parodo J, Taneja R and Marshall JC (2000) Phagocytosis of Candida albicans induces apoptosis of human neutrophils. Shock 14: 278-283

16. Salamone G, Giordano M, Trevani AS, Gamberale R, Vermeulen M, Schettinni $J$ and Geffner JR (2001) Promotion of neutrophil apoptosis by TNF- $\alpha$. J. Immunol. 166: 3476-3483

17. Jaye DL and Parkos CA (2000) Neutrophil migration across intestinal epithelium. Ann. N.Y. Acad. Sci. 915: 151-161

18. Wagner JG and Roth SA (2000) Neutrophil migration mechanisms, with an emphasis on pulmonary vasculature. Pharmacol. Rev. 52: 349-374

19. Watson RWG, Rotstein OD, Nathens AB, Parodo J and Marshall JC (1997) Neutrophil apoptosis is modulated by endothelial transmigration and adhesion molecule engagement. J. Immunol. 158: 945-953

20. Walzog B, Jeblonski F, Zakrzewicz A and Gaehtgens P (1997) $\beta 2$ integrins (CD11/CD18) promote apoptosis of human neutrophils. FASEB J. 11: $1177-1186$

21. Whitlock BB, Gardai S, Fadok V, Bratton D and Henson PM (2000) Differentia roles for $\alpha_{\mathrm{M}} \beta_{2}$ integrin clustering or activation in the control of apoptosis via regulation of Akt and ERK survival mechanisms. J. Cell Biol. 151: $1305-1320$
22. Nagata S (1997) Apoptosis by death factor. Cell 88: 355-365

23. Chen JJ, Sun Y and Nabel GJ (1998) Regulation of the proinflammatory effects of Fas ligand (CD95). Science 282: 1714-1717

24. Crispe IN (1994) Fatal interactions: Fas-induced apoptosis of mature T cells. Immunity 1: 347-355

25. Druilhe A, Cai Z, Haile S, Chouaib S and Prettolani M (1996) Fas-mediated apoptosis in cultured human eosinophils. Blood 87: 2822-2828

26. Um H, Orenstein JM and Wahl SM (1996) Fas mediates apoptosis in human monocytes by a reactive oxygen intermediate dependent pathway. J. Immunol. 156: 3469-3475

27. Liles WC, Kiener PA, Ledbetter JA, Aruffo A and Klebnoff SJ (1996) Differential expression of Fas (CD95) and Fas ligand on normal human phagocytes: implications for the regulation of apoptosis in neutrophils. J. Exp. Med. 184: 429-440

28. Fecho K and Cohen PL (1998) Fas ligand (gld)- and Fas (Ipr)-deficient mice do not show alterations in the extravasation or apoptosis of inflammatory neutrophils. J. Leukoc Biol. 64: 373-383

29. Villunger A, O'Reilly LA, Holler N, Adams J and Strasser A (2000) Fas ligand, $\mathrm{Bcl}-2$, granulocyte colony-stimulating factor, and p38 mitogen-activated protein kinase: regulators of distinct cell death and survival pathways in granulocytes. J. Exp. Med. 5: 647-658

30. Frasch SC, Nick JA, Fadok VA, Bratton DL, Worthen GS and Henson PM (1998) p38 Mitogen-activated protein kinase-dependent and -independent intracellular signal transduction pathways leading to apoptosis in human neutrophils. J. Biol. Chem. 273: 8389-8397

31. Aoshiba K, Yasui S, Hayashi M, Tamaoki J and Nagai A. (1999) Role of p38mitogen-activated protein kinase in spontaneous apoptosis of human neutrophils. J. Immunol. 162: 1692-1700

32. Alvarado-Kristensson M, Pörn-Ares Ml, Grethe $S$, Smith $D$, Zheng $L$ and Andersoson T (2002) p38 Mitogen-activated protein kinase and phosphatidyl 3kinase activities have opposite effects on human neutrophil apoptosis. FASEB J. 16: 129-131

33. Nolan B, Duffy A, Paquin L, De M, Collette H, Graziano CM and Bankey P (1999) Mitogen-activated protein kinases signal inhibition of apoptosis in lipopolysaccharide-stimulated neutrophils. Surgery 126: 406-412

34. Hofman P, Piche M, Farahi Far D, Le'Negrate G, Selva E, Landraud L, AllianaSchmid A, Boquet P and Rossi B (2000) Increased Escherichia coli phagocytosis in neutrophils that have transmigrated across a cultured intestinal epithelium. Infect. Immun. 68: 449-455

35. Kayagaki N, Kawasaki A, Ebata T, Ohmoto H, Ikeda S, Inoue S, Yoshino K, Okumura K and Yagita H (1995) Metalloproteinase-mediated release of human Fas ligand. J. Exp. Med. 182: 1777-1783

36. Xia Z, Dickens M, Raingeaud J, Davis RJ and Greenberg ME (1995) Opposite effects of ERK and JNK-p38 MAP kinases on apoptosis. Science 270: 13261331

37. Le'Negrate G, Selva E, Auberger P, Rossi B and Hofman P (2000) Sustained polymorphonuclear leukocytes transmigration induces apoptosis in intestinal epithelial T84 monolayers. J. Cell Biol. 150: 1479-1488

38. Watson R WG, Rotstein OD, Parodo J, Jimenez M, Soric I, Bitar R and Marshall JC (1997) Impaired apoptotic death signaling in signaling in inflammatory lung neutrophils is associated with decreased expression of interleukin-1beta converting enzyme family proteases (caspases). Surgery 122: $163-172$

39. Kim JM, Kim JS, Jung HC, Song IS and Kim CY (2000) Apoptosis of human gastric epithelial cells via caspase-3 activation in response to Helicobacter pylori infection: possible involvement of neutrophils through tumor necrosis factor alpha and soluble Fas ligands. Scand. J. Gastroenterol. 35: $40-48$

40. Takabatake N, Nakamura H, Inoue S, Terashita K, Yuhi H, Kato S, Yasumura S and Tomoike $H(2000)$ Circulating levels of soluble Fas ligand and soluble Fas in patients with chronic obstructive pulmonary disease. Respir. Med. 94: $1215-1220$

41. Hirata H, Takahashi A, Kobayashi S, Yonehara S, Sawai H, Okazaki T, Yamamoto K and Sasada M (1998) Caspases are activated in a branched protease cascade and control distinct downstream processes in Fas-induced apoptosis. J. Exp. Med. 187: 587-600

42. Slee EA, Adrain C and Martin SJ (2001) Executioner caspase-3, -6 and -7 perform distinct, non-redundant roles during the demolition phase of apoptosis. J. Biol. Chem. 276: 7320-7326 
43. Brannigan AE, O'Connell PR, Hurley H, O 'Neill A, Brady HR, Fitzpatrick JM and Watson RW (2000) Neutrophil apoptosis is delayed in patients with inflammatory bowel disease. Shock 13: 361-366

44. O 'Neill S, O 'Neill AJ, Conroy E, Brady HR, Fitzpatrick JM and Watson RW (2000) Altered caspase expression results in delayed neutrophil apoptosis in acute pancreatitis. J. Leukoc Biol. 68: 15-20

45. Pongracz J, Webb P, Wang K-Q, Deacon E, Lunn OJ and Lord JM (1999) Spontaneous neutrophil apoptosis involves caspase 3-mediated activation of PKC- $\delta$. J. Biol. Chem. 274: 37329-37334

46. Weiss SJ (1989) Tissue destruction by neutrophils. N. Engl. J. Med. 320: 365376
47. Parkos CA, Delp C, Arnaoult MA and Madara JL (1992) Neutrophil migration across a cultured intestinal epithelium: dependence on a CD11b/CD18mediated event and enhanced efficiency in the physiologic direction. J. Clin. Invest. 88: 1605-1612

48. Madara JL, Colgan S, Nusrat A, Delp C and Parkos C (1992) A simple approach to measurement of electrical parameters of cultured epithelial monolayers: use in assessing neutrophil-epithelial interaction. J. Tissue Culture Methods 14: 209-216

49. Nash S, Parkos C, Nusrat A, Delp C and Madara JL (1991) In vitro model of intestinal crypt abscess: a novel neutrophil-derived secretagogue (NDS) activity. J. Clin. Invest. 87: 1474-1477 